\title{
Spatial probability as an attentional cue in visual search
}

\author{
JOY J. GENG \\ Carnegie Mellon University and Center for the Neural Basis of Cognition, \\ Pittsburgh, Pennsylvania \\ and University College London, London, England \\ and \\ MARLENE BEHRMANN \\ Carnegie Mellon University and Center for the Neural Basis of Cognition, \\ Pittsburgh, Pennsylvania
}

\begin{abstract}
We investigated the role of spatial probabilities in target location during participants' performance of a visual search task. Experiments 1 and 2 demonstrated that spatial probabilities could serve as a powerful attentional bias that produced faster detection of targets in high-probability locations than of those in low- or random-probability locations. 'The effect could not be explained by repetition priming alone. Moreover, responses to targets in low-probability locations were slowed only when a distractor was present in the high-probability location. In Experiments 3-5, we compared the effects of spatial probability with an explicit endogenous cue and a salient exogenous cue. Facilitation due to spatial probability was independent of any benefit afforded by the explicit endogenous cue but interacted with the salient exogenous cue, such that the exogenous cue validity effect was compressed for targets in the high-probability location. Together, these results suggest that the spatial probabilities governing target location constitute a potent bias of visual processing and, as such, can be considered an attentional cue that differs from both typical explicit endogenous and salient exogenous cues.
\end{abstract}

Attention can be thought of as a bias that facilitates selection of relevant information from a world that contains more information than it is possible or desirable to process at once. The outcome of selection is likely the consequence of competitive interactions between various sources of information in the external environment and the goals internal to the observer: Attending to one location, object, or feature precludes, to some extent, the processing of others. The purpose of the experiments reported here was to examine how the probability of a target's appearance in a particular spatial location produces an attentional bias.

Within the domain of human visual processing, a number of studies have demonstrated that people are sensitive to regularities in the location of stimuli, such that targets in likely locations are detected more quickly than targets in less likely locations (see, e.g., Baker, Olson, \& Behrmann, 2004; Chun \& Jiang, 1998; Fiser \& Aslin, 2001; Geng \& Behrmann, 2002; Hoffmann \& Kunde, 1999; Hughes \&

This research was supported by an NSF IGERT training grant through the Center for the Neural Basis of Cognition and a Royal Society International Postdoctoral Fellowship to J.J.G. and by NIH Grant MH 54246 to M.B. We thank Roberta Klatzky and Árni Kristjánsson for useful comments on earlier drafts. We also thank the reviewers and editor for their constructive and insightful comments. Correspondence concerning this article should be addressed to J. J. Geng, Institute of Cognitive Neuroscience, University College London, 17 Queen Square, London WC1N 3AR, England (e-mail: j.geng@ucl.ac.uk).
Zimba, 1985; Kinchla, 1977; Kingstone \& Klein, 1991; Lambert \& Hockey, 1986; Miller, 1988; Posner, Snyder, \& Davidson, 1980; Reder, Weber, Shang, \& Vanyukov, 2003; Saffran, 2002; Shaw \& Shaw, 1977; Shomstein \& Yantis, 2004; Yantis \& Egeth, 1999). For example, Miller used a configuration of four horizontally aligned letters to test whether the probability of the target's location was coded in terms of absolute spatial location on the screen or in terms of relative position within the configuration. He found that performance was facilitated both when the target was located in the high-probability location defined by its position within the configuration and when it was located in relation to the screen (i.e., in a shifted position within the configuration). Participants also appeared to be sensitive to the likelihood that a specific stimulus form would be in a particular spatial location (Kingstone \& Klein, 1991; Lambert \& Hockey, 1986).

Using a similar paradigm, but with objectlike global configurations, Hoffmann and Kunde (1999) found greater facilitation for targets in likely locations defined by the configuration than for targets in likely locations defined by screen position. Chun and Jiang (1998) also reported facilitated target detection when a predictive probabilistic relationship existed between the target's location and the spatial configuration of distractor items. In their studies, the distractor configuration successfully cued the spatial location of visual search targets, even though multiple configuration and target associations were presented in 
parallel and predictive configurations were interleaved with nonpredictive configurations.

These studies provide clear evidence that implicitly presented spatial probabilities are a powerful determinant of visual processing. Few, however, have characterized these effects within the context of a standard attentional cue, which most often has been either a central cue (e.g., an arrow) or a salient event (e.g., a flash; see, e.g., Posner et al., 1980). One possible reason is that spatial regularities do not fit obviously within these two cue types, which have been characterized as top-down and bottom-up biases, respectively, where top-down generally refers to observer-directed sensory processing (e.g., search for a particular red-colored object), and bottom-up to the effect of salient information on processing (e.g., automatic orientation toward a flashing siren). Classification of probability effects as either top down or bottom up, however, is problematic. On the one hand, the anticipatory bias is similar to that induced by an explicit cue, but no symbolic interpretation is necessary and the effects are not under volitional control. On the other hand, probabilistic regularities are similar to exogenous cues in that the "cue" information is directly available in the stimulus, albeit over time, but also differ in that the cue information is not salient and does not capture attention.

One way to understand the role of spatial regularity as an attentional cue is to contrast its effects with those of other types of cues. However, to our knowledge, the only study that has compared implicit regularities with an attentional cue produced mixed results. Peterson and Kramer (2001a), using the contextual cuing paradigm of Chun and Jiang (1998), found reduced attentional capture by an exogenous event when the target was embedded within a predictive distractor context, as compared with an unpredictive context. This effect was obtained only when the first few blocks of training occurred without interference from the exogenous event and each trial was preceded by a masked version of the search display (i.e., the contextual cue; Peterson \& Kramer, 2001a, 2001b).

Unlike the relatively weak effect of the implicit contextual cue, however, explicit probabilistic cues have been found to minimize the effect of attentional capture by a stimulus onset, as well as by a color singleton (Yantis \& Egeth, 1999; Yantis \& Jonides, 1990). Interestingly, the degree to which the salient dimension interfered with target detection was commensurate with the predictive probability that each cue would be associated with the target. That is, a very predictive endogenous cue overrode the effect of a salient external cue, whereas a less predictive cue resulted in greater interference from the salient dimension (for more details on the interaction between explicit and salient external cues, see also Bisley \& Goldberg, 2003; Folk, Remington, \& Johnston, 1992; Hughes \& Zimba, 1985; Jonides, 1981; Müller \& Rabbitt, 1989; Theeuwes, Kramer, \& Atchley, 1999; Yantis, 1998; Yantis \& Jonides, 1990).

The primary questions addressed here concern how probabilistic regularities in the spatial location of a target bias visual processing and how any observed modulatory effect relates to existing concepts of endogenous and exogenous attentional cues. In order to address these questions, we first explored the dynamics of facilitation and inhibition induced by spatial probability within a visual search task. To anticipate our results in Experiments 1 and 2 , we found spatial probabilities to be a robust bias of visual attention, with facilitated processing of objects in likely locations and inhibited processing of objects in less likely locations. We then explored how spatial regularities compare with other attentional cues, in Experiments 3, 4, and 5 , by contrasting the probability cue against a standard explicit arrow cue and a salient exogenous flash. Again, to anticipate our results, we found that spatial probabilities interacted with salient exogenous events and were independent of, but qualitatively similar to, explicit endogenous cues.

\section{GENERAL METHOD}

Because the experiments used the same general method, we will describe it at the outset. Any additions or deviations from this are explained for the individual experiments. All the experiments were conducted using a 19-in. monitor in a dimly lit room. The participants were asked to place their heads in a chinrest that was $40 \mathrm{~cm}$ from the monitor. Each trial began with a fixation task, followed by a search display.

The search display consisted of orange $\mathrm{T}$ and $\mathrm{L}$ characters on a black background (see Figure 1). The T was always the target and appeared rotated $90^{\circ}$ to either the left or the right from upright. Each character subtended $1.5^{\circ}$ of visual angle at central fixation. All the characters were arranged in a circle, which subtended $13.3^{\circ}$ of visual angle. A target was present on every trial. The participants were instructed to press the " 1 " key on the number pad if the T was rotated to the left and the " 2 " key if it was rotated to the right. All the participants used the right hand to respond and were instructed to do so as quickly and accurately as possible.

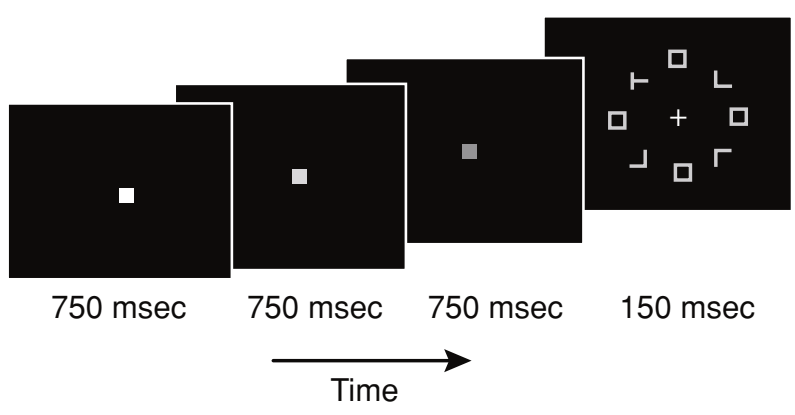

Figure 1. Example of trial procedure with fixation task and search display. Each fixation square was on for 750 msec or until response. The participants were instructed to press the space bar as soon as the square turned red in color and to withhold response otherwise. Immediately after response or a maximum of two color changes, the search display appeared for $150 \mathrm{msec}$. The participants responded manually to indicate whether the $T$ was rotated $90^{\circ}$ to the right or the left. 
In all the experiments, the manipulation of primary interest involved the uneven spatial distribution of targets across trials. Importantly, the probability manipulation of target location was orthogonal to the dimension of response (i.e., orientation discrimination). All the experiments consisted of uneven-probability blocks that were compared against random-probability blocks. In the uneven-probability blocks, the targets appeared in the high-probability location on $75 \%$ of the trials and were evenly divided among the low-probability locations on the remaining $25 \%$ of the trials. All the locations were equally likely (i.e., $25 \%$ ) to contain the target in random-probability blocks. No mention of the distribution of target locations was made at any stage of the experiment, nor was feedback given.

Eye tracking was employed in Experiment 1. Unless otherwise noted, maintenance of central fixation was monitored through performance on a second task in which the participants observed the color of a small square at fixation, which subtended approximately $0.9^{\circ}$ of visual angle. The fixation task occurred at the beginning of each trial and proceeded as follows. A colored square appeared in the center of the screen against a black background. The color of the square changed every $750 \mathrm{msec}$ for a maximum of two changes (three different colors). The participants were instructed to press the space bar with the left hand as quickly as possible if the square turned red in color. The square could turn red at any one of the three changes in color. Possible colors appeared in random order and were red, green, blue, aqua, pink, and yellow. Depression of the space bar was followed by the appearance of the search display for $150 \mathrm{msec}$. The participants were instructed to withhold response on the $10 \%$ of the trials in which the fixation square did not turn red. On these catch trials, the search display appeared automatically.

\section{EXPERIMENT 1}

The primary purpose of this initial experiment was to examine the effect of regularities in target location on performance in a visual search task. We were interested in whether implicit spatial probabilities might serve as a cue for perceptual selection. We compared performance on trials in which the target was in a high-, low-, or random(i.e., baseline) probability location. Faster and more accurate responses for targets in the high-probability location would suggest that spatial regularities produced an expectancy regarding the likely location of the target and that this expectation biased visual processing.

\section{Method}

Participants. Eighteen participants (15 of them female; $18-31$ years of age) participated. Twelve were Carnegie Mellon undergraduates, and 6 were recruited through the Institute of Cognitive Neuroscience at University College London. All the participants gave informed consent. One participant reported being left-handed. All had normal or corrected-to-normal vision. Data from 1 participant were excluded, due to inaccurate performance in the fixation task.

Design. The experiment consisted of four uneven-probability blocks and one random-probability block, each containing 180 trials. On every trial, the search display consisted of eight characters but only four possible target locations (see Figure 1). The four fillers lying along the vertical and horizontal meridians were always squares. Block ordering was random. Twelve participants engaged in the fixation task prior to the appearance of the search display, and 6 participants simply saw a fixation cross for $1,000 \mathrm{msec}$ prior to the search display onset. Eye movements for the latter 6 participants were monitored using an Applied Science Laboratories Model 504 remote eyetracker. Eye movements were sampled at $50 \mathrm{~Hz}$ and were monitored continuously throughout each trial.

\section{Results}

Data from the participants in the fixation task condition were excluded from further analyses if either a false alarm was made to a nontarget or the red target was missed. A trial was classified as a miss if there was no response within the 750-msec time period over which the target was present on the screen. One participant was excluded from further analyses, due to inaccurate performance on the fixation task. For all the remaining participants, misses and false alarms accounted for an average of $3 \%$ of the trials. Use of these criteria resulted in inclusion only of trials with rapid responses to the target $(M=442.3 \mathrm{msec}, S D=$ $36.8 \mathrm{msec}$ ), suggesting that the participants attended to the fixation detection task. The participants whose eye movements were tracked were also clearly able to maintain fixation (see Figure 2). There was a loss in signal from the eyetracker in approximately $5 \%$ of the data. An average of less than $1 \%$ of the data per participant was discarded due to fixations being outside a $2^{\circ}$ radius of visual angle from the fixation point during the $150-\mathrm{msec}$ stimulus display period.

Having established that the participants were maintaining central fixation, we now will report analyses of accuracy and mean response time (RT) data from each participant, using repeated measures ANOVA with the Bonferroni family-wise correction where appropriate. RTs exceeding 2,000 msec were discarded, which represented an average of less than $0.5 \%$ of the data per participant.

We first tested whether the between-participants factor of fixation (eye-tracking or fixation task) had a significant effect on performance. There was no main effect of fixation on accuracy or RT [accuracy, $F(1,16)=0.1$; RT, $F(1,16)=2.8]$. There was also no interaction between fixation and probability, using either measure [accuracy, $F(2,32)=0.1$; RT, $F(2,32)=0.04]$. In addition, we conducted separate ANOVAs for the two fixation conditions on the basic probability effect, using RTs. The effect was significant in both [eye tracking, $F(2,10)=21.8, M S_{\mathrm{e}}=$ $966, p<.001$; fixation task, $F(2,22)=28.8, M S_{\mathrm{e}}=$ $1,632, p<.001$; mean RTs (in milliseconds) for eyetracking and fixation tasks, respectively, random $=566.4$ and 512.9, high $=502.0$ and 441.1, and low $=620.4$ and 565.8]. These results demonstrated that performance on the search task was equivalent when the participants had their eyes monitored using a remote eye-tracking system and when they engaged in the fixation task. All the subsequent analyses were conducted on data collapsed across all 18 participants.

Probability and speed of target identification. The analysis of primary interest involved the main effect of 
Right Visual Field Targets
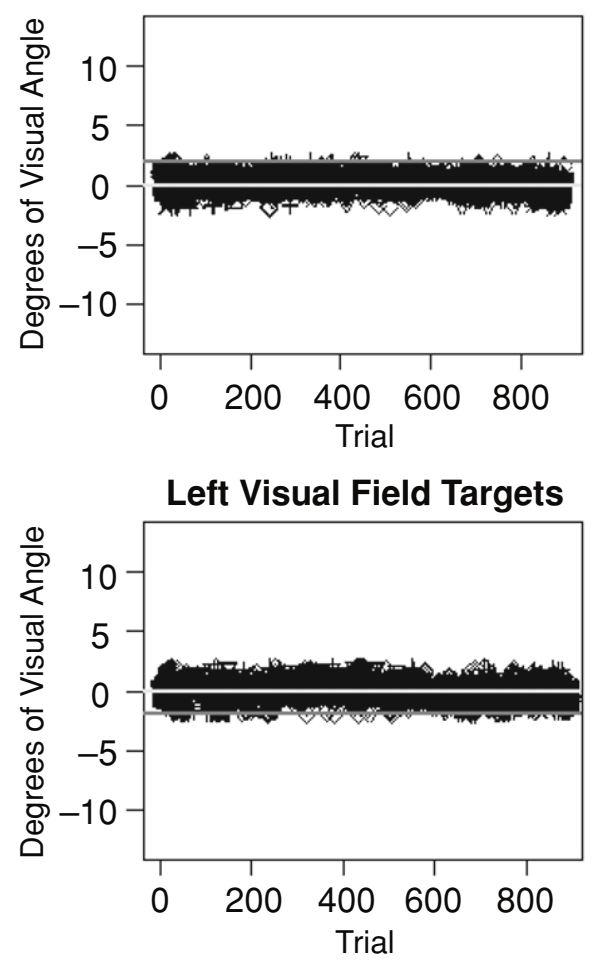

Figure 2. Eye movement data from 6 participants in Experiment 1 from all the trials included in the behavioral analyses and from which a signal was obtained from the eyetracker. Trials with eye position values outside a radius of $2^{\circ}$ of visual angle from the central fixation point were excluded from the analyses. For each trial, three data points were plotted per participant that represented three time-averaged bins across the 150 -msec duration search display. Ordinate values are in degrees of visual angle along the horizontal plane. The white line through the zero point is the aligned mean fixation point for all the participants, and the gray line marks $2^{\circ}$ from the center fixation point.

probability, and this was significant for both accuracy and RT [accuracy, $F(2,34)=8.15, M S_{\mathrm{e}}=0.001, p<.01$; RT, $F(2,34)=50.6, M S_{\mathrm{e}}=1,344, p<.001$; mean accuracy (in proportions) and RT (in milliseconds): random, .94 and 530.7; high, .96 and 461.4; low, .91 and 584.0]. Accuracy was greater for high-probability than for lowprobability targets (see Figure 3A), and responses to highprobability targets were significantly faster than those to random-probability targets, which were, in turn, faster than those for low-probability targets (see Figure 3B). These results confirmed our most basic expectation that the predictive spatial probability produced an attentional bias such that detection of targets in the high-probability location was facilitated, as compared with that of targets in other locations, whereas the opposite was true for targets in low-probability locations.

Probability and repetition priming. Although we attributed the speeded target identifications on the highprobability trials to the aggregated probability of the target's being in a particular location, facilitation may also have emerged from trial-to-trial repetition priming.
Studies that have not incorporated probabilistic regularities have shown that simple repetitions in target location significantly facilitate processing in subsequent trials (Hillstrom, 2000; Kristjánsson, Wang, \& Nakayama, 2002; Maljkovic \& Nakayama, 1996). In order to assess the relative contribution of probability, as compared with repetition priming from consecutive targets appearing in the same location, we conducted a post hoc test on the RT data, in which each trial was labeled as the $n$th trial, the immediately preceding trial as the 1-back trial, and the trial preceding that as the 2-back trial. The location of the target in each of the 1- and 2-back trials was coded as being in the same location as or a different location from the $n$th trial target.

Although we were interested in repetition priming effects for both high and low probability location targets, differences in the organization of the $n$-back trials for each probability condition necessitated separate analyses. First, multiple consecutive $\mathrm{S}$ trials (i.e., same 1-back and 2-back) occurred only for high- and random-probability locations. The occurrence of more than two consecutive targets in the same low-probability location was improbable. Second, there were three different low-probability locations in our visual search display, resulting in two classes of "different" 1-back trials: high-probability 1-back trials $\left(\right.$ different $\left._{\text {high }}\right)$ and different low-probability 1-back trials (different ${ }_{l o w}$ ). This was in contrast to 1-back trials for highprobability trials, all of which were in a low-probability location. Due to these differences, we used the high- and randomprobability trials to assess the effect of same-location repetition priming and the low-probability trials to assess the effect of different-location, nonrepetition inhibition.

If spatial regularities modulated behavior solely through trial-to-trial repetition priming, there should be equal response facilitation for repetitions in a location, regardless of the probability of the target's being in that location. If, however, probabilities produced an expectation for the upcoming target's location over and above that of repetition priming alone, the magnitude of facilitation from the local repetition should be greater for targets in high-probability locations than for those in random-probability locations. This comparison served as the critical test of whether our manipulation was solely an outcome of repetition priming or whether it produced additional attentional biasing.

We conducted a $2 \times 2 \times 2$ ANOVA involving $n$th trial probability (high or random), 1-back-trial location, and 2-back-trial location. Using accuracy data, only the main effect of 1-back location was significant $[F(1,17)=15.9$, $\left.M S_{\mathrm{e}}=0.002, p<.001\right]$. Using RT data, the main effects of 1-back and 2-back target locations were significant $\left[1\right.$-back, $F(1,17)=68.6, M S_{\mathrm{e}}=4,066, p<.001 ; 2$-back, $\left.F(1,17)=20.0, M S_{\mathrm{e}}=1,284, p<.001\right]$, as were the $n$th trial probability $\times 1$-back location and $n$th trial probability $\times 2$-back interactions $\left[F(1,17)=23.9, M S_{\mathrm{e}}=730.5\right.$, $\left.p<.001 ; F(1,17)=5.5, M S_{\mathrm{e}}=1,569.1, p<.05\right]$. Tukey's HSD post hoc test was used to compare means within the interactions. The $n$th trial probability $\times 1$-back location interaction consisted of a significant difference between high-same and random-same, but no difference 

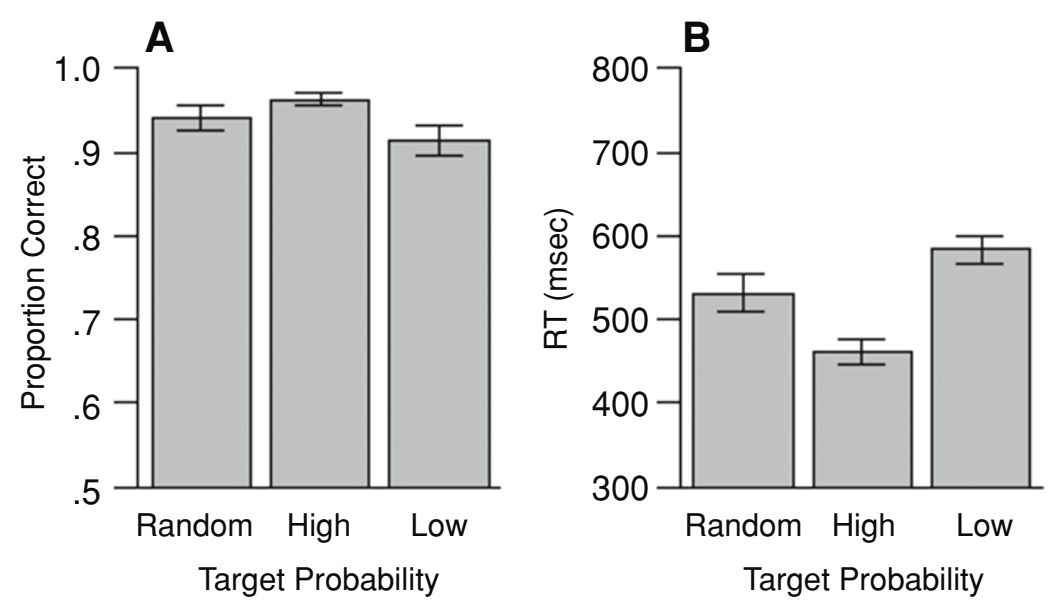

Figure 3. The basic effect of spatial regularity in Experiment 1. (A) Proportion of correct responses was greater for high- than for low-probability trials. (B) Reaction times (RTs) to targets appearing in the high-probability location were significantly shorter than those to targets in both the random- and the low-probability locations.

between high-different and random-different. The differences between same and different in both the high- and random-probability conditions were also significant (see Figure 4A). That is, consistent with the previously demonstrated presence of location repetition priming, responses to targets in the same location as the immediately preceding target were facilitated regardless of location probability, but additionally, the magnitude of repetition facilitation was greater for targets in the high-probability location. Similarly, the one-way ANOVA of 1-back trials for low-probability location targets was significant $\left[F(2,34)=20.9, M S_{\mathrm{e}}=\right.$ $1,907, p<.001]$, and involved repetition priming when the previous target was in the same location and no difference between trials in which the preceding target was in a highor a different low-probability location.

The significant interaction between the $n$th trial probability and the 2-back location was driven by a differ- ence between high-same and high-different and no difference between random-same and random-different (see Figure 4B). Importantly, this result suggested that the location of the 2-back target had an effect in the highprobability condition, but not in the random-probability location. Repetition priming for random-location targets did not extend back beyond the immediately preceding trial. The three-way interaction between $n$th trial probability, 1-back location, and 2-back location was not significant $[F(1,17)=0.3],{ }^{1}$ and neither was the interaction between 1-back and 2-back trials $[F(1,17)=0.5]$.

\section{Discussion}

The primary result from this experiment was faster and more accurate responses for targets in the high-probability location than for targets in low- or random-probability locations. This result indicated that probability served as
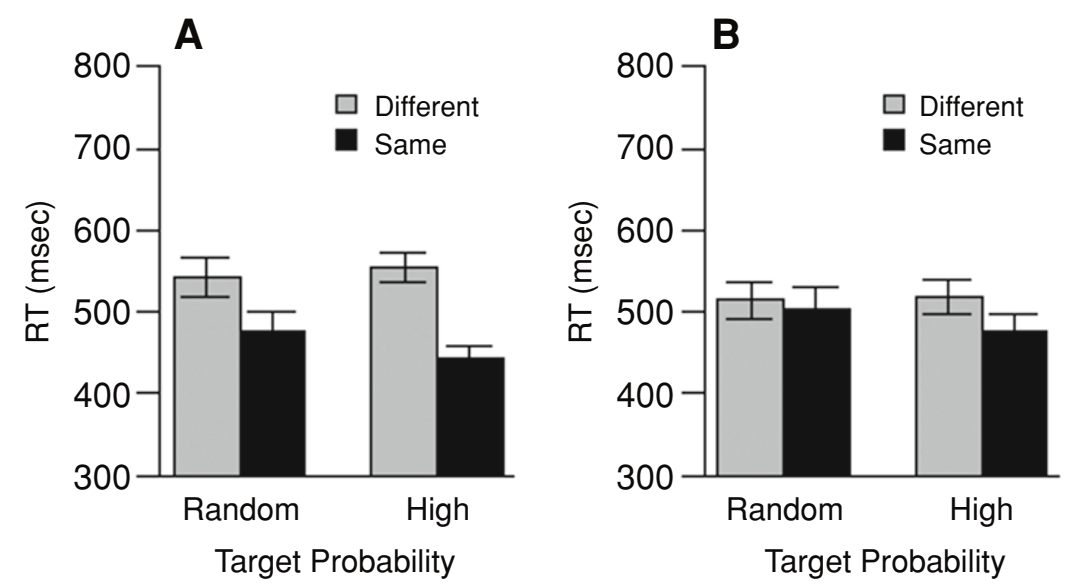

Figure 4. (A) Data from the $n$th trial $\times$ 1-back interaction in Experiment 1. Greater facilitation was present for same-location repetitions in high- than in randomprobability target locations. (B) Data from the $n$th trial $\times 2$-back interaction in Experiment 1. Significant facilitation for same-location trials was present for high- but not for random-probability location targets. 
an effective attentional cue, even when the location designated as high probability changed over the course of the experiment and the participants were given no information regarding the spatial probability of targets. Moreover, the effect of probability cannot simply be explained by spatial repetition priming: Although immediate repetition priming was present for targets in all locations, there was additional facilitation for targets in the high-probability location.

Repetition priming was present in all the probability conditions, but there was greater sensitivity to both 1-back and 2-back repetitions in the high-probability compared to the random-probability location. The patterns of the interactions suggested the presence of facilitation for high probability location targets over and above that of spatial repetition priming alone. It is likely that the effect of spatial probability emerges from repetition priming and that they involve the same or similar mechanisms. This would be unsurprising, since the statistic of probability that we used was indistinguishable from frequency. It is interesting, however, that the effect of repetition priming for random-probability targets in this experiment was restricted to the 1-back trial, whereas previously reported experiments have shown longer lasting effects. This fact, in conjunction with the finding that the three-way interaction in this experiment was not significant, suggested that the result of greater facilitation in the high-probability location was not simply due to likelihood of the $2+$ back trials being in the same location. We are unable to account for these differences in the duration of repetition priming other than to note that our experimental design and manipulation differed from those in previous experiments in a number of ways. For example, whereas the task in previous experiments often has involved search for an object possessing a particular feature, our defining and reported features were always fixed and independent of the dimension of repetition. Therefore, under the conditions of our experiment, it is clear that the spatial probability effect we have described cannot simply be subsumed under that of repetition priming, and we refer to this additional effect as probability cuing, rather than as repetition priming.

\section{EXPERIMENT 2}

Experiment 1 established probability as an effective processing bias that facilitated detection of targets in likely locations and inhibited detection of targets in less likely locations. The purpose of Experiment 2 was to extend those findings by investigating the conditions that produce facilitation and inhibition. In order to do so, we used two distractor set sizes (four and eight; see Figure 5A for the eight-item display) and two configurations of the fouritem displays (Figures 5B and 5C). This design allowed us to examine the strength of facilitation in a standard visual search paradigm with varying set size, as well as the inhibitory effect of objects in the high-probability location on low-probability targets. Both aspects are critical to understanding the overall effect of spatial probability as an attentional cue.

First, increasing the number of visually similar distractors usually results in a monotonic increase in RT (and/or a decrease in accuracy). The change in performance reflects the necessity for serial search mechanisms to locate the target and is taken as an indication of the difficulty of the search process. The likelihood of finding a given target on the first search cycle is inversely related to the total number of items in the visual field, and the mean search time for a single target approximates the time it takes to process half of the items present (Desimone \& Duncan, 1995; Duncan \& Humphreys, 1989; Treisman \& Gelade, 1980; Zelinsky, Rao, Hayhoe, \& Ballard, 1997). The fact that high-probability targets were detected more quickly than low-probability targets in Experiment 1, however, suggested that the spatial probability effectively biased attention toward objects in the high-probability location. Consequently, we expected that high probability location targets would show less of an increase in RT between set sizes (i.e., a shallower slope), since interference from distractors is reduced for cued objects. However, because the spatial probability was not entirely predictive (i.e., only $75 \%$ predictive), some additional processing time associated with that uncertainty was expected for eight-item displays, as compared with four-item ones (Bricolo, Giane-
A) Set size eight

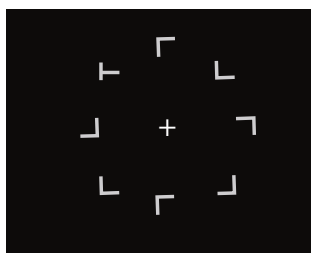

B) Set size four: $\operatorname{low}_{\mathrm{h}}$

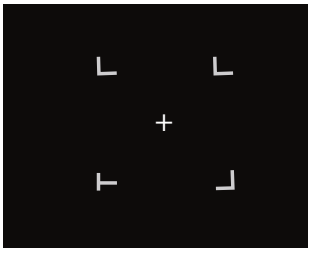

C) Set size four: low $_{0}$

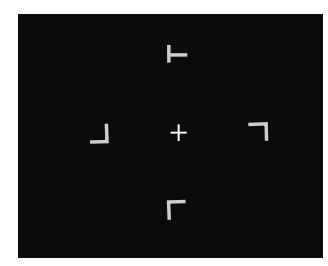

Figure 5. Examples of the three possible search display configurations used in Experiment 2. (A) Set size eight display in which the target is in the highprobability location in the top left corner. (B) Set size four display in which the target was in a low-probability location and a distractor was present in the high-probability location (i.e., low $_{h}$ trials). (C) Set size four display in which the target was in a low-probability location and no distractor was present in the high-probability location (i.e., low $_{0}$ trials). 
sini, Fanini, Bundesen, \& Chelazzi, 2002; Tse, Sheinberg, \& Logothetis, 2003; Zelinsky, 1999). The change in performance between set size as a function of target location probability reflected the degree to which the probability cue was successful in facilitating detection of the target and overriding competition from distractor items.

Second, by using two different configurations of fouritem displays, we were able to assess the effect of a distractor in the high-probability location on detection of a low-probability location target. That is, we compared lowprobability target trials in which the high-probability location contained a distractor $\left(\operatorname{low}_{h}\right)$ and trials in which it was empty $\left(\operatorname{low}_{0}\right)$. If the participants developed a strong expectation that targets would appear in the high-probability location in advance of the stimulus display (e.g., an anticipatory spotlight of attention), one would expect slower performance on low-probability targets, regardless of whether or not a distractor object was actually in the high-probability location $\left(\mathrm{RT} \operatorname{low}_{\mathrm{h}}=\mathrm{RT} \mathrm{low}_{0}\right)$. Such a spatial priming effect would be comparable to a purely spatial cue that directed attention to a particular location in space in advance of the visual search display (e.g., an explicit arrow or a salient exogenous event). Alternatively, if inhibition of low probability location targets was due to interference from the increased processing efficiency or priority of objects in the highprobability location, slowed responses should occur only when a distractor was present in the high-probability location (e.g., RT $\operatorname{low}_{\mathrm{h}}>\mathrm{RT} \mathrm{low}_{0}$ ). When no distractor was in that location, no interference would occur, and RTs should be as short as those in the random-probability condition.

\section{Method}

Participants. Sixteen Carnegie Mellon University undergraduate students participated for course credit (4 of them female; 18-24 years of age). All had normal or corrected-to-normal vision. All the participants gave informed consent. Two participants reported being left-handed. Data from 1 participant were excluded, due to inaccurate performance on the fixation task.

Design. Displays with a set size of eight consisted of one target $\mathrm{T}$ stimulus and seven distractor $\mathrm{L}$ stimuli evenly distributed around a circle. Displays with four items appeared in one of two configurations, each containing one target $\mathrm{T}$ and three distractor $\mathrm{L}$ stimuli. Two configurations were used to equate the total frequency of stimuli in each location across the two set sizes and to compare performance between configurations in which the high-probability location contained a distractor $\left(\operatorname{low}_{\mathrm{h}}\right)$ and those in which the high-probability location was empty $\left(\mathrm{low}_{0}\right)$. The presentations of all the configurations and set sizes were randomly interleaved (see Figure 5).

An additional 50-msec interstimulus interval (ISI) between the final fixation square and the appearance of the search display was inserted in this experiment to reduce the difficulty of the eight-item trials. The additional 50-msec ISI was inserted into the set size of four trials as well, in order to equate the duration of the trials in the two conditions. There were eight uneven probability blocks (each of the eight locations was designated the high-probability location once) and one random-probability block. Each block consisted of 112 trials. Uneven probability blocks consisted of 84 high-probability trials and 28 low-probability trials. Block ordering was random. A total of 1,008 trials were run over the course of the experiment. There was no obvious delineation between blocks. Two self-terminating breaks were provided at even intervals. No information regarding the location manipulation was given to the participants in advance.

\section{Results}

As before, trials in which there was either a false alarm or a miss during the fixation task were discarded. Data from 1 participant were excluded, due to inaccurate performance. For the remaining 15 participants, false alarms and misses accounted for an average of $7.7 \%$ trials per participant. A miss occurred if there was no response within the 750-msec time period over which the target was present on the screen. Furthermore, responses to correctly detected targets were $\operatorname{rapid}(M=455.7 \mathrm{msec}, S D=$ $36.7 \mathrm{msec}$ ). We will report analyses of accuracy and mean RT data from each participant below, using repeated measures ANOVA with the Bonferroni family-wise correction of paired comparisons where appropriate. In the RT analyses, values exceeding $2,000 \mathrm{msec}$ were discarded. This represented an average of less than $1.3 \%$ of the data per participant.

Effect of set size on high-probability targets. To test the cost of additional distractors on response to targets in the different probability conditions, we conducted a repeated measures ANOVA with the within-participants factors of probability (high, low, or random) and set size (four or eight). Both main effects and the interaction were significant, using accuracy [probability, $F(2,28)=33.4$, $M S_{\mathrm{e}}=0.001, p<.001$; set size, $F(1,14)=61.1, M S_{\mathrm{e}}=$ $0.002, p<.001$; interaction, $F(2,28)=17.4, M S_{\mathrm{e}}=$ $0.001, p<.001$ ] and RT [probability, $F(2,28)=109.9$, $M S_{\mathrm{e}}=3,072, p<.001$; set size, $F(1,14)=165.0, M S_{\mathrm{e}}=$ $1,976, p<.001$; interaction, $F(2,28)=37.4, M S_{\mathrm{e}}=743$, $p<.001$ ] data (see Figures 6A and 6B). In addition, using the Bonferroni correction for multiple comparisons, there was a significant stepwise difference between the high-, low-, and random-probability conditions in the accuracy and RT data (mean accuracy [in proportions correct] and RT [in milliseconds]: random, .91 and $565.0 \mathrm{msec}$; high, .96 and $418.3 \mathrm{msec}$; low, .88 and $624.4 \mathrm{msec}$ ) and shorter RTs for set sizes of four than for set sizes of eight (four, .95 and $475.7 \mathrm{msec}$; eight, .88 and $596.0 \mathrm{msec}$ ).

Although RTs were longer for a set size of eight than for a set size of four in all probability conditions, the magnitude of difference was clearly dissimilar, as is indicated by the significant interaction. This was confirmed in a one-way ANOVA on the difference scores between set sizes (set size of eight - set size of four) for each probability condition [accuracy, $F(2,28)=17.4, M S_{\mathrm{e}}=0.002, p<.001$; RT, $\left.F(2,28)=37.4, M S_{\mathrm{e}}=1,485, p<.001\right]$. The highprobability location had a significantly smaller value, as compared with both random- and low-probability locations, with both measures. The difference between random and low probability was not significant [means of (set size of eight - set size of four): random, .10 and $142.0 \mathrm{msec}$; high, .01 and $51.7 \mathrm{msec}$; low, .09 and $167.4 \mathrm{msec}$ ). The smaller cost of additional distractors in the high-probability location can be thought of either as less inhibition from each of the additional distractors (i.e., interference amounting to an average of $71 \mathrm{msec} /$ additional distractor in the random condition, as compared with $25.9 \mathrm{msec}$ for highprobability targets) or as increased search efficiency (i.e., 
A

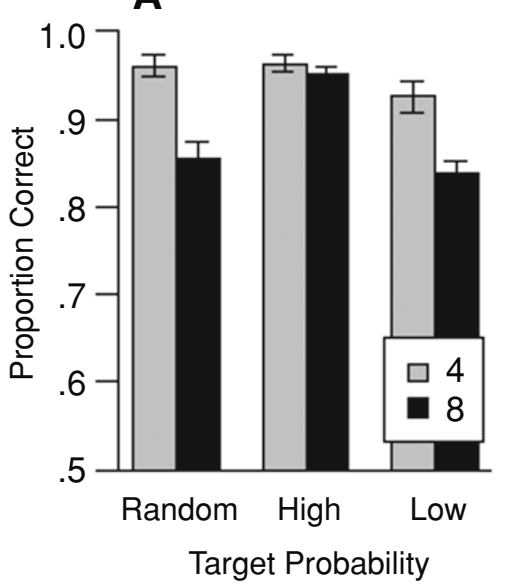

B

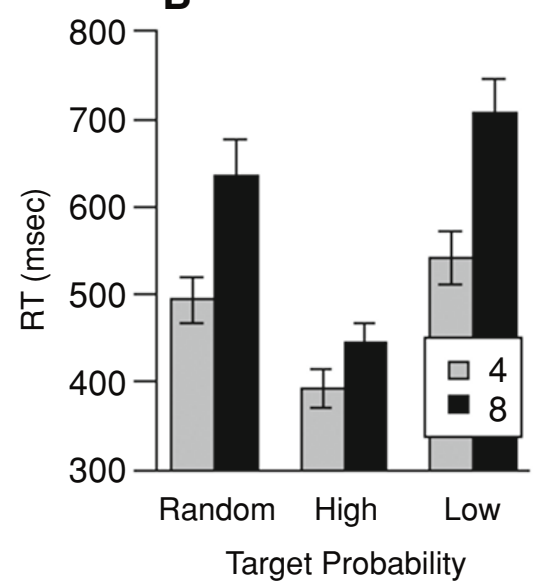

Figure 6. Interaction between $n$th trial target probability (random, high, or low) and display set size (four or eight) for (A) accuracy and (B) response time (RT) data. Both behavioral measures show less interference between set sizes for high- than for random- and low-probability targets.

the likelihood of attending to 2 additional distractors in the random condition, as compared with only 0.4 additional distractors, on average, in the high-probability condition).

Targets in four-item displays. Because two different set size of four configurations were used, low-probability targets appeared in displays in which the high-probability location either contained a distractor or was empty low $_{\mathrm{h}}$ or low $_{0}$ ). These two different configurations provided some leverage for understanding the effect of the probability manipulation on low-probability targets. Accuracy and RT data from the possible four-item conditions (low ${ }_{\mathrm{h}}, \mathrm{low}_{0}$, high, or random) were entered into a oneway ANOVA. The main effect was significant, using both measures [accuracy, $F(3,42)=5.0, M S_{\mathrm{e}}=0.002, p<$ .005 ; RT, $F(3,42)=57.9, M S_{\mathrm{e}}=1,398, p<.001$; mean proportion correct and RT (in milliseconds): random, .96 and 494.0; high, .96 and 392.4; $\operatorname{low}_{\mathrm{h}}, .91$ and 565.1; $\operatorname{low}_{0}$, .93 and 522.7]. Importantly, in the RT data, responses in the low $_{\mathrm{h}}$ trials were significantly slower than those in the low $_{0}$ and the random trials $[t(14)=5.1, p<.0005$; $t(14)=4.3, p<.0005]$, but there was no significant difference between the $\operatorname{low}_{0}$ and the random trials $[t(14)=$ $2.0, p=.07]$. None of the differences in accuracy survived Bonferroni correction. The presence of a distractor in the high-probability location inhibited processing of a simultaneously present low-probability target, but when no distractor was present in the high-probability location, inhibition was reduced (see Figure 7). This result is consistent with the idea that the processing of the highprobability location object interfered with the processing of objects in other locations, rather than pure spatial priming of the high-probability location prior to display onset. That is, the probability manipulation had an effect on processing only through a conjunction of object and spatial information.

\section{Discussion}

The purpose of this experiment was to explore how the probability bias we identified in Experiment 1 facilitated and inhibited the processing of objects in different locations. First, we found that interference from increased numbers of distractors was reduced significantly for high probability location targets. Second, using the two displays with set sizes of four to compare responses to low-probability trials, we found that inhibition of low-probability targets occurred only when a distractor was present in the high-probability location. On trials in which no distractor was in that location, RTs were

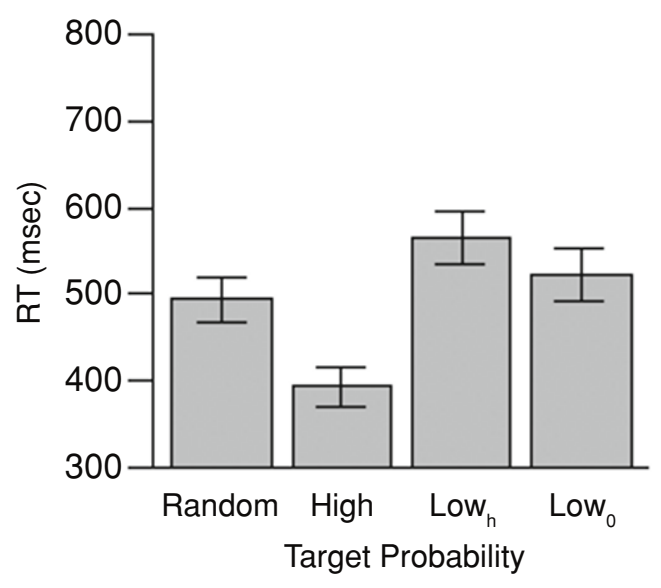

Figure 7. Data from trials with a display set size of four. Responses to high probability target trials were fastest. Responses to $\operatorname{low}_{h}$ trials were slower than those to $\operatorname{low}_{0}$ and random trials, but $\operatorname{low}_{0}$ trials were equivalent to random trials. The results suggested that the presence of a distractor in the high-probability location was necessary for interference to occur for low probability location targets. 
equivalent to those in the random-probability block. This suggested that the spatial regularity facilitated processing of the object in the high-probability location, rather than producing pure spatial priming per se.

Another possible explanation for the finding that there was inhibition for the displays with a set size of four only with a distractor in the high-probability location involves contextual cuing (Chun \& Jiang, 1998; Hoffmann $\&$ Kunde, 1999). It may be that over the course of a biased probability block, the participants learned that the high-probability location was associated only with the set size of eight and a particular set size of four configuration. However, since presentation of our displays was very rapid and the consistent cue allowed for predisplay expectancies to play a role, further experiments will be necessary to disentangle how global spatial expectancies interact with context effects. Nevertheless, it is clear that processing of high probability location objects was facilitated, resulting in faster detection of targets in that location and slower detection of targets when a distractor or no object occupied that location.

Together, Experiments 1 and 2 demonstrated that the presence of a spatial probability altered patterns of visual search and suggested that spatial probability operated as an attentional cue. However, the effects were neither obviously top down, in the typical sense of an explicit symbolic cue driving attentional selection, nor obviously bottom up, since the "cue" information was not salient and did not capture attention. Rather, we interpreted the effects as being a consequence of the high probability location object's having a competitive processing advantage, as compared with objects in the other locations. This competitive bias resulted in facilitated performance for the "cued" object. In the following experiments, we attempted to understand the effects of spatial probability within the context of more traditional characterizations of attention by contrasting it directly with an explicit cue and then with a salient exogenous cue.

\section{EXPERIMENT 3}

The previous results suggested that the spatial probability cue may have operated differently from an explicit spatial cue. In this experiment, we tested this hypothesis by pairing the implicitly presented spatial regularity with an explicit arrow cue within the same paradigm. The presence of two attentional cues could result in one cue's masking the effect of the other entirely (i.e., additivity) or in an interaction between the two. Complete masking of one cue by another would occur if the two cues were tracked within completely overlapping processing systems. In this case, a validity effect would be observed for only one cue. Additivity would suggest some separation in the processing of each cue type, which could involve either one or two systems. Finally, an interaction would suggest that the two cues were represented within interrelated systems or subsystems. If the cues were equal in strength, performance would be optimal with two valid cues, poor with two invalid cues, and intermediate with one valid and one invalid cue. If one cue was dominant, however, performance would be facilitated whenever the stronger cue was valid, and the effect of the weaker cue would emerge only when the dominant cue was invalid.

\section{Method}

Participants. Twenty-six participants (12 of them females; ranging in age from 18 to 30 years) were recruited from the Carnegie Mellon University community. Each person was either given course credit or paid $\$ 5$ for his or her participation. The experiment took approximately $40 \mathrm{~min}$ to complete. All the participants gave informed consent. All the participants had normal or corrected-to-normal vision, and 4 reported being left-handed. Data from 3 participants were excluded, since their accuracy fell below $75 \%$ correct in more than one condition of interest.

Design. The experiment consisted of six blocks. Each block consisted of 100 trials. Three of the six blocks were combined-cue blocks in which both the probability cue and the arrow cue were $70 \%$ valid. The predictive validity of the cues was reduced, as compared with previous experiments, in order to ensure a sufficient number of trials in each experimental condition. The breakdown of the number of trials in each of those three blocks was as follows: 49 trials in which both probability and the arrow were valid, 21 trials in which only the arrow was valid, 21 trials in which only probability was valid, and 9 trials in which neither cue type was valid. In each of the three blocks, a different location was designated the highprobability location. One block was a probability-alone block in which the probability cue was $70 \%$ valid and the arrow was neutral (noninformative). One block was an arrow-alone block in which the arrow cue was $70 \%$ valid and the targets appeared randomly in all possible locations. Finally, there was one baseline block in which the targets appeared randomly in all locations and the arrow cue was neutral. All the blocks were randomly interleaved for each participant. There were four possible target locations, and for each participant, a different combination of three was included in the joint probability and arrow condition, and the fourth was used for the probability-only block. A mandatory self-terminating rest period occurred after every two blocks.

On each trial, the fixation task was followed by the appearance of an arrow, which was then followed by the search display. The participants were told simply that the arrow would be informative most of the time, but not all of the time. No information was given regarding the probability cue. The fixation task in this experiment involved a maximum of one color change, instead of two. The arrow cue, in predictive blocks, pointed to one of the four possible target locations. Neutral arrows had the appearance of all four arrows overlaid on top of each other and were clearly uninformative. The arrow cue was visible for $400 \mathrm{msec}$, followed by an ISI during which a fixation cross was present on an otherwise blank screen. To ensure that the arrow stimulus onset asynchrony (SOA) we chose was maximally effective, we included two ISI values ( 250 and $1,000 \mathrm{msec}$ ) between the arrow and the search display. ISI was a between-participants factor.

\section{Results}

As before, trials in which there was either a false alarm or a miss during the fixation task were discarded. False alarms and misses accounted for $7.9 \%$ of the trials per participant. A miss occurred if there was no response within the 750-msec time period over which the target was present on the screen. Furthermore, responses to correctly detected targets were rapid $(M=453.8 \mathrm{msec}, S D=$ $41.9 \mathrm{msec}$ ). We will report analyses of accuracy and mean RT data from each participant below, using repeated measures ANOVA with the Bonferroni family-wise correc- 
tion of paired comparisons where appropriate. In the RT analyses, values exceeding 2,000 msec were discarded. This represented an average of less than $2 \%$ of the data per participant.

First, we conducted an analysis to test whether the between-participants factor of ISI had a significant effect on performance. ISI ( 250 or $1,000 \mathrm{msec}$ ) was entered into a mixed-factor ANOVA with probability (random, high, or low) and arrow validity (neutral, valid, or invalid) as factors. The main effect of ISI was not significant using accuracy data $[F(1,21)=0.6]$ but was significant using RT data $\left[F(1,21)=5.2, M S_{\mathrm{e}}=157,952, p<.05\right]$. ISI did not, however, interact significantly with either probability $[F(2,42)=0.1]$ or arrow validity $[F(2,42)=0.7]$. Since ISI did not contribute significantly to the comparisons of interest, we collapsed across the two ISI durations in the remaining analyses.

The purpose of this experiment was to compare strength of probability with an explicit arrow as biases of visual attention. Therefore, the primary analysis of interest involved the factors of probability (random, high, or low) and arrow (neutral, valid, or invalid). Both main effects of probability and arrow were significant for accuracy as well as RT, but the interactions were not [for accuracy, probability, $F(2,44)=4.8, M S_{\mathrm{e}}=0.007, p<.05$; arrow, $F(2,44)=$ 5.0, $M S_{\mathrm{e}}=0.005, p<.05$; interaction, $F(4,88)=0.5$; or for RT, probability, $F(2,44)=35.3, M S_{\mathrm{e}}=7,033, p<.001$; arrow, $F(2,44)=18.7, M S_{\mathrm{e}}=11,287, p<.001$; interaction, $F(4,88)=0.5]$. This suggested that the cues operated independently of each other (see Figures 8A and 8B).

Within the main effect of spatial probability, significant differences were found between high and low probability for both accuracy and RT and between high and random probability for RT only. There was no significant difference between low and random probability for either accuracy or RT (mean accuracy [in proportion correct] and RT [in milliseconds]: random, .93 and 574.3; high,
.95 and 491.2; low, .91 and 607.8). For the arrow cue, significant differences were found between valid and invalid conditions for both accuracy and RT and between neutral and invalid conditions in RT only. There was no difference between neutral and valid arrow cues (mean accuracy [in proportions] and RT [in milliseconds]: neutral, .93 and 544.9; valid, .95 and 510.1; invalid, .91 and 618.3). These results indicated that the basic effect of each cue operated as expected but that probability produced relatively greater processing facilitation of objects in the high-probability location, whereas the arrow produced relatively greater inhibition for invalidly cued locations in this experiment.

\section{Discussion}

The effect of the probability and arrow cues were additive. Whether or not there are some conditions under which these two cues would interact remains an interesting question, one that would require parametric manipulations of the conditions adopted here. It is possible, for example, that when the predictive probabilities associated with each cue are unequal, one may dominate the other. However, under the conditions of this experiment, in which the properties of the two cues were as similar as possible, each cue exerted an effect on visual processing such that a valid/high-probability cue resulted in faster and more accurate responses than did an invalid/ low-probability cue. That they were independent in this experiment and that the pattern of pairwise comparisons within each condition differed suggested that the two cues involved distinguishable processes. One possibility is that the two cue types are processed at different levels within the same endogenous attentional system. For example, it may be that the explicit arrow cue produced a top-down volitional signal that resulted in selection of the cued spatial location, whereas the spatial probability modulated the bottom-up processing efficiency of objects in the most probable location (Experiment 2). (Note that the terms
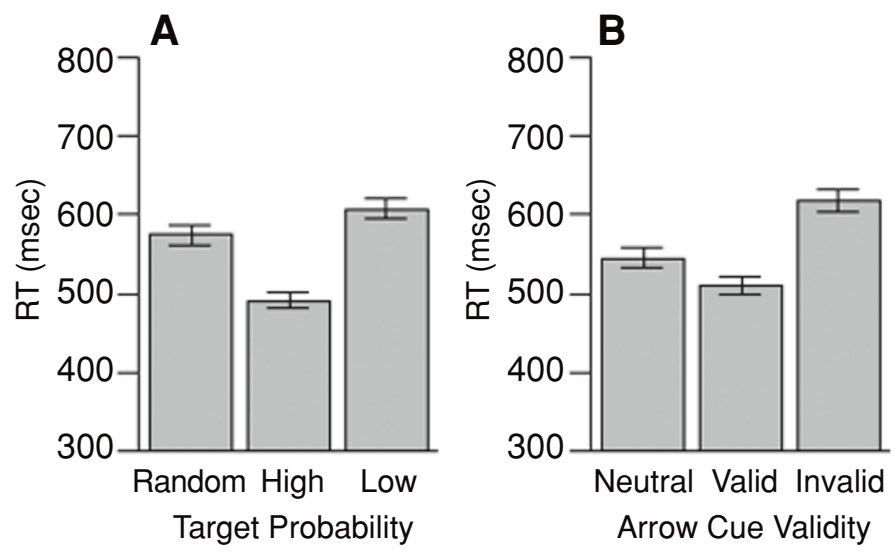

Figure 8. Mean response times (RTs) in Experiment 3. (A) Responses to high probability location targets were significantly different from those from both random- and low-probability targets, which were not different from each other. (B) Responses to targets preceded by an invalid arrow cue were slower than those to either valid or neutral arrow cues, which were not different from each other. 
top-down and bottom-up refer to the inferred cognitive processes involved, rather than to a distinction based on neural feedback and feedforward processing.) These two levels of processing may be related to the level of awareness participants have in learning and exploiting the cues. Both of these processes may co-occur within the same endogenous attentional system, but because the facilitation occurred at different points in processing, independent effects were observed. Although completely independent mechanisms can also give rise to additive effects, a shared endogenous system also predicts that probability and explicit arrows will generate similar patterns of interaction when paired with an exogenous cue, whereas separate systems would not necessarily predict such similarities. We examined this possibility in Experiments 4 and 5.

\section{EXPERIMENT 4A}

In Experiment 3, we found that spatial probability and the arrow cue produced independent effects. It was unclear, however, whether the spatial probability could be characterized as an endogenous cue that operated within the same processing stream as the explicit arrow cue. In this experiment, we examined the relationship between the spatial probability and a salient exogenous event. In addition, the comparison also provided an indirect way of assessing the similarity of the spatial probability to an endogenous explicit cue. Although there has been limited evidence relating probabilistic information to exogenous events (Peterson \& Kramer, 2001a, 2001b), there has been substantial evidence demarcating the relationship between explicit endogenous cues and salient exogenous events.

For example, in one such study, Müller and Rabbitt (1989) asked observers to make a decision regarding a target $\mathrm{T}$ that could appear in one of four locations within a display. At variable SOAs preceding the target array, a predictive arrow cue appeared, and one of the possible target boxes briefly brightened. At short SOAs, responses were more accurate when the target was at the brightened location, but at longer SOAs, response was more accurate when the target was at the location indicated by the arrow. That is, at short SOAs, the transient brightening interrupted voluntary orientation of attention, but the obligatory capture dissipated rapidly. Moreover, the strength of the capture was modified by its correspondence with the endogenous cue, such that responses were most accurate when the exogenous event occurred at the location indicated by the arrow cue. These and other, similar results have led investigators to conclude that spatial orienting can be driven by either an automatic or a voluntary mechanism and that the two are separate but interactive (Bisley \& Goldberg, 2003; Corbetta \& Shulman, 2002; Folk et al., 1992; Hughes \& Zimba, 1985; Theeuwes, 1991; Yantis \& Jonides, 1990). Thus, finding a strong interaction between spatial probability and an exogenous event that is similar to that between explicit cues and exogenous events would bolster the suggestion made in Experiment 3 that spatial probabilities operate within the same endogenous atten- tional system as explicit cues, but perhaps at a different level of processing.

\section{Method}

Participants. All the participants were given course credit in exchange for their participation, and all had normal or corrected-tonormal vision. Data from 1 participant were excluded for being less than $75 \%$ accurate in more than one condition of interest. Data from 16 participants were included in the reported analyses ( 13 of them female, 18-26 years of age). All the participants gave informed consent. One participant reported being left-handed.

Design. Each trial proceeded as follows. A fixation cross appeared at the center of the screen. After 1,000 msec, an exogenous cue appeared at one of the four possible target locations for $50 \mathrm{msec}$, followed immediately by the search display. The exogenous cue was composed of an $\mathrm{x}$ sign subtending approximately $0.5^{\circ}$ of visual angle. Each search display character subtended $1^{\circ}$ of visual angle at central fixation. The experiment had a $3 \times 3$ design. There were three probability levels (random, high, or low) and three levels of exogenous cue (absent, valid, or invalid). The location of the exogenous cue was always randomly distributed $(20 \%$ in each of four target locations and $20 \%$ absent). The participants were told that the exogenous cue was informative. As in all the other experiments, no mention of the probability cue was made. For each block of 60 trials, the target was in the high-probability location on 45 trials, 9 of which were preceded by a valid cue, 27 of which were preceded by an invalid cue in one of the three other possible target locations, and 9 in which the exogenous cue was absent. In the 15 low-probability trials, the exogenous cue was valid on 3 trials, invalid on 9 trials, and absent on 3 trials. In random-probability blocks, both the target and the exogenous cue appeared in all locations with equal frequency. Note that in this experiment, there were only 60 trials per block, the stimuli were presented on a 15 -in. monitor, and no fixation task was included at the start of each trial. All other aspects of the design were identical to the conditions described previously.

\section{Results}

As before, RT values exceeding 2,000 msec (an average of less than $1 \%$ of the data per participant) were excluded from the RT analyses, and Bonferroni correction for paired comparisons was used where appropriate. The data were entered into a probability (random, high, or low) $\times$ exogenous event (absent, valid, or invalid) repeated measures ANOVA. In order to replicate the probability effect, as well as the classic exogenous cue effect, we looked first at the main effects. As in Experiment 1, there was a significant effect of probability for both accuracy and RT [accuracy, $F(2,30)=11.9, M S_{\mathrm{e}}=0.0007, p<.001$; RT, $\left.F(2,30)=79.7, M S_{\mathrm{e}}=2,360, p<.001\right]$. The participants were more accurate for high- than for low-probability targets and faster for high-probability targets than for both random- and low-probability targets. Responses to random targets were significantly more accurate and faster than those to low-probability targets (mean accuracy [in proportions] and RT [in milliseconds]: random, .976 and 506.3; high, .982 and 437.1; low, .957 and 562.0). The main effect of exogenous event was also significant in accuracy and RT [accuracy, $F(2,30)=3.2, M S_{\mathrm{e}}=0.0004$, $p=.05$; RT, $\left.F(2,30)=33.6, M S_{\mathrm{e}}=1,011, p<.001\right)$. Consistent with known effects, responses were most accurate and fastest when the cue was valid, less accurate and slower when it was invalid, and intermediate when it 
was absent (mean accuracy [in proportions] and RT [in milliseconds]: absent, .97 and 505.6; valid, .98 and 473.5; invalid, .97 and 526.4). All three comparisons were significant using RT, and only the difference between valid and invalid was significant using accuracy data. The main effects bore out the expected pattern of facilitation and inhibition in response to each cue type.

Consistent with the idea that spatial probabilities operate similarly to explicit endogenous cues, the interaction between probability and the exogenous event was significant in the RT data $\left[F(4,60)=4.2, M S_{\mathrm{e}}=391.9, p<\right.$ .01 ; see Figure 9]. Significant differences between means within the interaction were determined using Tukey's HSD post hoc correction. As is evident in Figure 9, the interaction was driven by the difference between exogenous cue levels within each probability condition. (All three probability conditions were significantly different from each other within each exogenous cue condition and, thus, simply reflected the main effect of probability.) When the target was in either a random- or a low-probability location, there was a significant difference between the valid and the absent trials and no difference between the absent and the invalid trials. When the target was in the high-probability location, however, there was no significant difference between the valid and the absent trials or between the absent and the invalid trials. The effect of the exogenous cue, however, was not eliminated in the high-probability location: RTs were significantly shorter in the valid than in the invalid trials in all three probability locations, although the magnitude of the normalized RT difference [(invalid - valid)/(invalid + valid)] was smaller in the high- than in the random-probability condition $\left[F(2,30)=4.3, M S_{\mathrm{e}}=0.0006, p<.05\right.$; difference values: random, .065; high, .039; low, .056]. This pattern suggested that the response facilitation normally afforded by the exogenous cue was diminished when the target was in the high-probability location.

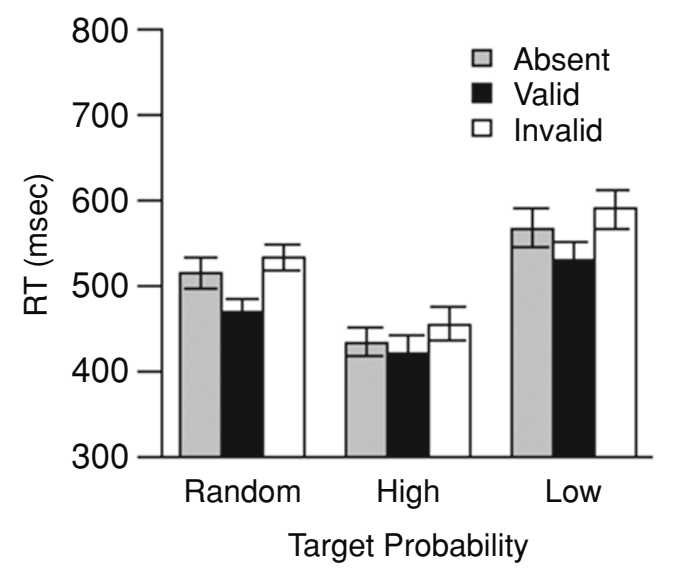

Figure 9. Interaction between probability and exogenous cue in Experiment 4A. The effect of the exogenous cue was compressed for high probability location targets but was present in low and random locations, suggesting that the effect of probability was relatively stronger than that of the exogenous event.

\section{Discussion}

In this experiment, we contrasted spatial probability with an exogenous event. We found that facilitation due to a valid exogenous event was reduced when the target was in the high-probability location, as compared with either a random- or a low-probability location. On the other hand, responses to objects in the high-probability location were faster than those to targets elsewhere, regardless of where the exogenous cue occurred, although there was still a significant difference between valid and invalid exogenous events for targets in the high-probability location. This interaction indicated that the two sources of information competed against each other when they cued different locations but that probability was the stronger determiner of selection outcome. When the target was not in the highprobability location, the effect of the exogenous cue was clearly visible.

We interpreted these results to suggest not that sensitivity to the exogenous event was inhibited per se but, rather, that the probability cue was a stronger source of bias. As such, objects in that location were more likely to win the competition for selection. Nevertheless, the contribution of the exogenous cue to the competition was present and had a more visible influence on behavior when the target was not in the high-probability location. This result is consistent with the findings in Peterson and Kramer (2001a), in which the implicit contextual cue reduced attentional capture by an exogenous event only under specific training and viewing conditions. Clearly, the probability in our experiment was much simpler than that of the Chun and Jiang (1998) contextual cuing manipulation used by Peterson and Kramer (2001a), and this difference may well account for the difference in strength of the probability cue relative to the exogenous cue.

In addition, these data mirror previous comparisons of explicit cues with salient exogenous events well (Yantis \& Egeth, 1999; Yantis \& Jonides, 1990) and support our previous suggestion that probability operates within a shared endogenous processing stream with explicit probabilistic cues. However, an alternative explanation that we have not yet considered for the compression between valid, neutral, and invalid trials in the high-probability location involves a response floor effect. That is, RTs might have been shorter when the exogenous cue preceded the target in the high-probability location if not for a limitation in response speed. We addressed this concern in the next experiment.

\section{EXPERIMENT 4B}

In Experiment 4A, we observed a compression of RTs in the high-probability location. In this experiment, we tested whether the effects we found were due to a response floor effect by increasing the predictive validity of the probability cue. The design of this experiment was identical to that in Experiment $4 \mathrm{~A}$, with the exception that the probability cue was now $100 \%$, instead of $75 \%$, predictive. If the RT compression was produced by a floor effect, the results of this experiment should be very similar to those 
in the previous experiment. However, if the compression was due to the predictive strength of the probability cue, we would expect shorter RTs in the high-probability location, as well as greater compression of the exogenous cue effect in Experiment 4B than in Experiment 4A. This result would obtain if the ability of the exogenous cue to compete decreases as the predictive strength of the probability cue increases. Note that we have selected 100\% probability, rather than a more intermediate value (e.g., $90 \%$ ), to fully uncover the extent to which probability enhances performance. We were interested in exhibiting the maximal benefit possible so that we might conclude that the compression observed previously could not possibly have been the result of a floor effect.

\section{Method}

Participants. Ten members of the Carnegie Mellon University community ( 7 of them female; $20-32$ years of age) participated for either course credit or $\$ 5$. All had normal or corrected-to-normal vision. All the participants gave informed consent. Two participants reported being left-handed.

Design. The design of this experiment was identical to that in Experiment 4A, with the exception that the probability cue was now $100 \%$ valid, rather than $75 \%$. This resulted in only two probability conditions: high and random.

\section{Results}

Because the low-probability condition was precluded by the design of the experiment, all comparisons included only the high- and random-probability conditions. RTs exceeding 2,000 msec were excluded, which involved less than $1 \%$ of the data per participant. The main effect of exogenous cue was significant only in the RT data [accuracy, $F(2,18)=0.25$; RT, $F(2,18)=6.3, M S_{\mathrm{e}}=$ $528.5, p<.01]$. The main effect of probability was significant for both accuracy and RT [accuracy, $F(1,9)=$ $18.5, M S_{\mathrm{e}}=0.0017, p<.01$; RT, $F(1,9)=70.1, M S_{\mathrm{e}}=$ $9,537, p<.001]$. The interaction between probability and exogenous cue was also significant only for RT [accuracy, $F(2,18)=0.6$; RT, $\left.F(2,18)=5.7, M S_{\mathrm{e}}=449.9, p<.05\right]$. The interaction resulted from a significant difference between the valid and the invalid exogenous cue conditions in the random-probability condition that was absent in the high-probability condition. As with Experiment 4A, we found shorter RTs and greater compression of RTs over exogenous cue validity for targets in the high-probability location, as compared with those in the random-probability locations.

As a test of whether the previous results were a consequence of a floor effect, the data from Experiments 4A and $4 \mathrm{~B}$ were entered into a mixed-factor repeated measures ANOVA with the between-participants factor of experiment $(75 \%$ or $100 \%)$ and the within-participants factors of probability (random or high) and exogenous cue (absent, valid, or invalid). Since RTs were more informative in the individual analyses in each experiment, only the RT data were entered into the analysis. The main effect of experiment on RT was not significant $[F(1,24)=0.8]$, but it interacted with probability $\left[F(1,24)=30.6, M S_{\mathrm{e}}=\right.$ 4,693, $p<.001]$.
The experiment $\times$ probability interaction clearly indicated that RTs in the $100 \%$ experiment were shorter for high probability location targets but longer for randomprobability targets, as compared with the $75 \%$ experiment (mean RTs [in milliseconds]: 100\%-random $=568.79$, $100 \%$-high $=357.73,75 \%$-random $=506.25,75 \%$-high $=$ 437.12). RTs to high-probability targets when the probability cue was $100 \%$ predictive were shorter than when the cue was $75 \%$ predictive, demonstrating that the RTs in the $75 \%$ experiment were not due to a floor effect. Moreover, this result suggested that the more predictive the probability cue was, the more effective it was in biasing visual processing toward objects in the high-probability location.

\section{Discussion}

It is clear from the comparison of Experiments $4 \mathrm{~A}$ and $4 \mathrm{~B}$ that the results from the previous experiment were not due to a response floor effect. RTs were shorter in the high-probability location when the probability cue was $100 \%$ predictive than when it was only $75 \%$ predictive. In addition, the results indicated that the competitive strength of the probability bias was graded by the predictive power of the cue. Increasing the cue predictiveness further reduced the normal facilitation and inhibition effects of the exogenous cue. It is possible that the shorter RTs in Experiment $4 \mathrm{~B}$ were due to the fact that the participants were more likely to have been aware of the predictive probability than were those in Experiment 4A. Like other tasks in which structural regularities are implicitly embedded within the stimuli, awareness of the regularities appears to vary along a continuum, and RT has been found to correlate with this continuum of awareness (Frensch \& Runger, 2003; Keele, Ivry, Mayr, Hazeltine, \& Heuer, 2003).

\section{EXPERIMENT 5}

In Experiment 3, we found that probability and an explicit arrow cue had independent effects on behavior. We hypothesized that the two cues engaged the same endogenous system but that the arrow cue produced a topdown spatial bias, whereas the probability cue resulted in a bottom-up change in the processing efficiency of objects in the high-probability location. The different level of processing that each cue acted on may be related to the level of awareness the participants had in using the cue. If both probability and arrow cuing engage the same system, we might expect them to interact with an exogenous cue, which is thought to involve a different subsystem, in qualitatively similar ways. On the other hand, because one requires volitional shifts of attention, whereas the other involves the tracking of spatial regularities, they may differ quantitatively in terms of the degree to which they affect behavior. Experiment 4 suggested that the interaction of an exogenous event with spatial probability was similar to that with an explicit arrow cue, but because we could only qualitatively compare our results with others from the literature, it was impossible to draw any conclusions regarding the relative strength of spatial probabilities and explicit arrows as endogenous cues. In this experiment, 
we attempted to gain a measure of the relative strength of the probability and the arrow cues by contrasting each against an exogenous event within the same experiment. Although indirect, the result would be indicative of the effectiveness of each endogenous cue in determining the outcome of attentional selection.

\section{Method}

Participants. Twenty-three undergraduate Carnegie Mellon University students participated for course credit ( 8 of them female; 18-24 years of age). Two participants reported being left-handed. Three participants were excluded because their accuracy fell below $75 \%$ correct in more than one condition of interest. All the participants gave informed consent.

Design. In this experiment, both the probability and the arrow cue conditions were labeled endogenous, and the following factors were included: endogenous cue (random, high probability, low probability, valid arrow, or invalid arrow), exogenous cue (absent, valid, or invalid), and exogenous cue SOA (50 or $100 \mathrm{msec}$ ). We used two exogenous cue SOAs, both of which have been shown to be within the range in which an exogenous cue is most effective (Posner, 1980). Separate blocks were run for the probability and arrow conditions. A total of 920 trials were included. The display materials and task were identical to those in the previous experiment, except that the timing was altered to accommodate the longer SOA necessary for an explicit arrow cue to be effective. Below, we will describe the trial procedure separately for blocks in which the endogenous cue was probability and for those in which it was an arrow.

Probability cue trials proceeded as follows. A fixation cross appeared for either 1,050 or $1,000 \mathrm{msec}$, followed by a 50 -msec exogenous cue. Either immediately following or after another $50 \mathrm{msec}$ of fixation, the search display appeared for $150 \mathrm{msec}$. The screen then went blank until the participant responded. The total duration of the trial was the same regardless of whether the exogenous cue SOA was 50 or $100 \mathrm{msec}$. The only difference was in whether a $50-\mathrm{msec}$ fixation occurred before or after the exogenous cue.

Arrow cue trials proceeded as follows. A fixation cross appeared for $1,000 \mathrm{msec}$, followed by the arrow cue for $300 \mathrm{msec}$. Immediately thereafter, the fixation screen reappeared for 150 or $100 \mathrm{msec}$. The exogenous cue then appeared for $50 \mathrm{msec}$, followed either by another $50-\mathrm{msec}$ fixation or immediately by the search display for $150 \mathrm{msec}$. The screen then went blank until the participant responded. The SOA between the arrow and the search display was $600 \mathrm{msec}$. As in the probability trials, the two exogenous SOA trial procedures were identical, except for the duration of the fixation screen immediately before and after the exogenous cue. Again, the participants were told that the arrow cue would be informative most of the time, but not all of the time.

\section{Results}

All analyses were conducted using repeated measures ANOVA with Bonferroni correction where appropriate. RTs exceeding 2,000 msec were excluded. This represented an average of less than $1 \%$ of the data per participant. The first analysis addressed the question of whether the duration of the exogenous cue SOA affected performance. For this comparison, the factors of endogenous cue, exogenous cue, and SOA (50 or $100 \mathrm{msec}$ ) were entered into an analysis. The main effect of SOA was not significant for accuracy $[F(1,19)=2.3]$ but was for RT $\left[F(1,19)=21.4, M S_{\mathrm{e}}=16,204, p<.001\right]$. RTs were shorter, overall, with an SOA of $100 \mathrm{msec}$ than with one of $50 \mathrm{msec}(100,526.3 \mathrm{msec} ; 50,574.3 \mathrm{msec})$. Because SOA did not interact with the factors of interest, we collapsed across the two SOA durations in the remaining analyses.

The main analysis of interest involved the factors of endogenous cue (random, high probability, low probability, valid arrow, or invalid arrow) and exogenous cue (absent, valid, or invalid). Both the main effects and the interaction were significant. The main effects confirmed our basic expectations about the efficacy of each cue. First, the main effect of endogenous cue [accuracy, $F(4,76)=$ $3.5, M S_{\mathrm{e}}=0.0018, p<.05$; RT, $F(4,76)=22.3, M S_{\mathrm{e}}=$ $19,138, p<.001]$ involved significant differences in RT between the three probability conditions, which replicated the main effect of probability, and a significant difference between the valid and the invalid arrow conditions, which replicated the basic cue validity effect. Second, the main effect of exogenous cue was significant only in the RT data and involved significant differences between all three levels $\left[F(2,38)=37.8, M S_{\mathrm{e}}=2,459, p<.001\right]$.

The interaction was driven primarily by a compression of the exogenous cue conditions in the high-probability and valid arrow conditions, as compared with their lowprobability and invalid arrow counterparts [accuracy, $F(8,152)=2.5, M S_{\mathrm{e}}=0.0009, p<.05 ;$ RT, $F(8,152)=$ $5.8, M S_{\mathrm{e}}=1,286, p<.001$; see Figure 10]. This result was similar to the results in Experiments 4A and 4B. Using Tukey's HSD post hoc correction on the RT data, we found a similar pattern of results for the high-probability and the valid arrow conditions: There was no significant difference between any of the three exogenous cue conditions. Correspondingly, in both the low-probability and the invalid arrow conditions, there were significant differences between the valid and the invalid exogenous cue conditions. In addition, in each of the three exogenous cue levels within the high-probability condition, responses were faster than in their valid arrow counterparts, suggesting a baseline advantage for high-probability locations (difference score, valid arrow - high probability) for each exogenous cue condition (in milliseconds): absent, 58.1; valid, 59.9; invalid, 62.0.

\section{Discussion}

When targets appeared in the location predicted by either spatial probability or the explicit arrow cue, the normal exogenous cue validity effect was reduced. This suggested that both cues biased the participants toward the most likely location for the target to appear. However, RTs were shorter in each of the three high-probability conditions, as compared with their valid arrow counterparts, suggesting that there was an overall advantage for the probability cue over the arrow cue. This effect cannot simply be attributed to shorter RTs in the probability blocks than in the arrow blocks, since all but one of the low-probability and invalid arrow conditions were statistically equivalent. The quantitative differences between the probability and the arrow conditions is consistent with our hypothesis in Experiment 3 that explicit arrow and implicit spatial probabilities may engage the same endogenous system but, perhaps, operate at different levels of processing. On the basis of the present data, it is not pos- 


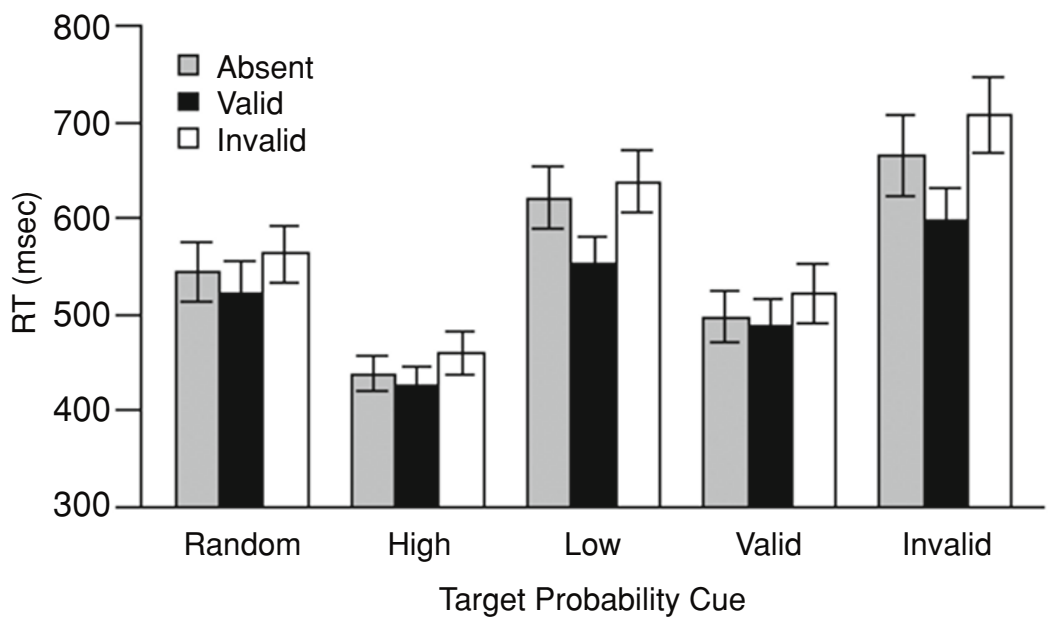

Figure 10. Interaction between endogenous and exogenous cues in Experiment 5. An exogenous cue validity effect was present in random, low-probability, and invalid arrow cue conditions. There were similar patterns of RT compression in the highprobability and valid-arrow endogenous conditions but, overall, shorter RTs in the high-probability condition than in the valid arrow condition.

sible to determine whether our hypothesis is correct or not. Nevertheless, that probability and arrow cues operate qualitatively the same but produce quantitative differences in behavior suggests that they may bias visual processing through a shared system.

\section{GENERAL DISCUSSION}

The purpose of these experiments was to better understand the effect of probabilistic spatial regularities on visual processing. Although the effect of spatial probabilities on stimulus structure has been explored within the visual domain previously, it has rarely been described in terms of its relationship to classic visual attentional paradigms involving explicit or exogenous cues. Perhaps one reason for this is that probabilistic information does not fit easily within the categorization of attentional cuing effects as being either top-down and volitional or bottom-up and salient. In Experiments 1 and 2, we first established that spatial probability produced changes in behavior similar to those resulting from attentional cuing in visual search, and in Experiments 3-5, we compared the probability bias with standard attentional cues, in order to place it within the context of attentional effects.

In Experiment 1, we established that visual processing was biased toward objects in the high-probability location and that the magnitude of facilitation was greater than that attributable to repetition priming alone. In Experiment 2, we explored in greater detail the patterns of facilitation and inhibition produced by the spatial probability. Consistent with the facilitation effects in Experiment 1, we found that increasing the number of distractors had a smaller effect on high-probability targets than on either random- or low-probability targets. Interestingly, however, low probability location targets were inhibited only when a distractor could appear in the high-probability location. These results suggested that the mechanism under- lying the effect of spatial probability involved increased processing efficiency of targets in the high-probability location. Faster processing would provide objects in the high-probability location with a competitive advantage for selection and would give rise to inhibition of other, simultaneously present objects. When there is no object in the high-probability location, however, no object would have a processing advantage, and therefore, no additional inhibition would ensue for objects displayed in low-probability locations.

In Experiments 3, 4, and 5, we explored the effect of spatial probability as a type of attentional cue by contrasting its effects with those of an explicit arrow cue and a salient exogenous event. The spatial probability and the arrow cue produced independent effects, suggesting some separation in processing. In contrast, spatial probability interacted with the exogenous flash such that the degree to which the exogenous cue validity effect was suppressed was commensurate with the predictive strength of the probability cue. This result was consistent with previous findings demonstrating a continuum between the predictive probability of explicit cues and attentional capture and suggested that implicit and explicit probabilistic cues may bias attention through similar mechanisms (e.g., Patel \& Sathian, 2000; Yantis \& Egeth, 1999; Yantis \& Jonides, 1990). However, there were quantitative differences between the explicit arrow and the implicitly presented spatial probability cues, such that the latter produced greater facilitation overall, suggesting that under these conditions, spatial probability was a stronger bias on visual processing.

Together, these results led us to hypothesize that the probability cue operated through a conjunction of spatial and object information and was processed within a system shared by explicit spatial cues. However, it is possible that whereas the explicit spatial cue operated through strategic top-down anticipation of an object in the cued spatial 
location, the spatial probability cue may have operated through bottom-up changes in the processing efficiency of stimuli in the probable location. This might result in the two cues' differing in time course and generalizability of learning. These differences may involve priming based on different memory mechanisms or attentional modulation at different levels within the visual system and, therefore, may correlate with participants' awareness of the cue.

There is good reason to believe that the mechanisms underlying learning of the probability cue are related to mechanisms involved in repetition priming. This seems likely both because of the rapid time course in which behavior begins to reflect sensitivity to the probability cue and because of the fact that our spatial probability manipulation is based on simple frequencies that reflect a cumulative measure of events stored in memory. Previous studies of repetition priming and probability cuing have speculated that implicit short-term or episodic memory mechanisms may be involved in the representing of repetitions (Chun \& Jiang, 1998; Hillstrom, 2000; Hoffmann \& Kunde, 1999; Huang, Holcombe, \& Pashler, 2004; Kristjánsson et al., 2002; Maljkovic \& Nakayama, 2000), and our findings are consistent with these ideas. We note, of course, that repetition priming itself is a subset of a large family of forms of priming, ranging from iconic to short-term and to long-term priming. Exactly where endogenous cues and spatial probabilities have their effects on visual processing along this priming continuum remains to be specified, but documenting the similarities and differences between them in their time course, specificity, and history (strength of trace) deserves further investigation and discussion.

Although we did not have a quantifiable measure of awareness during the experiments, we did ask the participants, at the end of the experiment, whether or not they had noticed that targets were more likely to appear in a particular location. We found great variability in the degree of awareness and ability to report the content of that awareness. There also appeared to be less awareness associated with experiments in which there were multiple cues, suggesting that the harder the task, the less the participants were aware of the spatial regularity. This is consistent with findings in which learning of regularities interacted with attentional load (Musen, 1996) and the idea that implicit learning mechanisms may give rise to explicit awareness (Willingham, Salidis, \& Gabrieli, 2002).

The relationship between learning mechanisms involved in repetition priming, simple probabilities, and explicit attentional cuing warrants further research. Particularly, it would be interesting to pursue the suggestion from these experiments that a probabilistic regularity represents an intermediate level of attentional cuing that lies between repetition priming and explicit volitional cues. Attentional biases certainly lie on a continuum of potency and awareness, and these experiments attempted to bridge two ends of a spectrum that appeared to engage different mechanisms but produced similar behavioral effects. Consistent with the ideas of biased competition and integrated competition (Desimone \& Duncan, 1995; Duncan, Hum- phreys, \& Ward, 1997), spatial probabilities appear to be another means by which visual processing can be biased toward some information at the cost of not processing other information. From this perspective, attentional effects emerge from various stimulus and goal-oriented factors that modulate processing at a number of levels, rather than necessarily being strictly top down or bottom up. Probabilistic spatial regularities can easily be understood as an attentional bias within such a framework.

\section{REFERENCES}

Baker, C., Olson, C., \& Behrmann, M. (2004). Role of attention and perceptual grouping in visual statistical learning. Psychological Science, 15, 460-466.

Bisley, J. W., \& Goldberg, M. E. (2003). Neuronal activity in the lateral intraparietal area and spatial attention. Science, 299, 81-86.

Bricolo, E., Gianesini, T., Fanini, A., Bundesen, C., \& Chelazzi, L. (2002). Serial attention mechanisms in visual search: A direct behavioral demonstration. Journal of Cognitive Neuroscience, 14, 980-993.

Chun, M. M., \& JiAng, Y. (1998). Contextual cueing: Implicit learning and memory of visual context guides spatial attention. Cognitive Psychology, 36, 28-71.

Corbetta, M., \& Shulman, G. L. (2002). Control of goal-directed and stimulus-driven attention in the brain. Nature Reviews Neuroscience, 3, 201-215.

Desimone, R., \& Duncan, J. (1995). Neural mechanisms of selective visual attention. Annual Review of Neuroscience, 18, 193-222.

Duncan, J., \& Humphreys, G. W. (1989). Visual search and stimulus similarity. Psychological Review, 96, 433-458.

Duncan, J., Humphreys, G. [W.], \& Ward, R. (1997). Competitive brain activity in visual attention. Current Opinion in Neurobiology, 7, 255-261.

FISER, J., \& ASLIN, R. N. (2001). Unsupervised statistical learning of higher-order spatial structures from visual scenes. Psychological Science, 12, 499-504.

FolK, C. L., Remington, R. W., \& Johnston, J. C. (1992). Involuntary covert orienting is contingent on attentional control settings. Journal of Experimental Psychology: Human Perception \& Performance, 18, 1030-1044.

Frensch, P. A., \& Runger, D. (2003). Implicit learning. Current Directions in Psychological Science, 12, 13-18.

Geng, J. J., \& Behrmann, M. (2002). Probability cueing of target location facilitates visual search implicitly in normal participants and patients with hemispatial neglect. Psychological Science, 13, 520-525.

Hillstrom, A. P. (2000). Repetition effects in visual search. Perception \& Psychophysics, 62, 800-817.

Hoffmann, J., \& Kunde, W. (1999). Location-specific target expectancies in visual search. Journal of Experimental Psychology: Human Perception \& Performance, 25, 1127-1141.

Huang, L., Holcombe, A. O., \& Pashler, H. (2004). Repetition priming in visual search: Episodic retrieval, not feature priming. Memory \& Cognition, 32, 12-20.

Hughes, H. C., \& ZimbA, L. D. (1985). Spatial maps of directed visual attention. Journal of Experimental Psychology: Human Perception \& Performance, 11, 409-430.

JoNIDES, J. (1981). Voluntary versus automatic control over the mind's eye's movement. In J. [B.] Long \& A. [D.] Baddeley (Eds.), Attention and performance $I X$ (pp. 187-203). Hillsdale, NJ: Erlbaum.

Keele, S. W., Ivry, R., Mayr, U., Hazeltine, E., \& Heuer, H. (2003). The cognitive and neural architecture of sequence representation. Psychological Review, 110, 316-339.

KINCHLA, R. A. (1977). The role of structural redundancy in the perception of visual targets. Perception \& Psychophysics, 22, 19-30.

Kingstone, A., \& Klein, R. (1991). Combining shape and position expectancies: Hierarchical processing and selective inhibition. Journal of Experimental Psychology: Human Perception \& Performance, 17, 512-519.

Kristuánsson, Á., Wang, D., \& Nakayama, K. (2002). The role of priming in conjunctive visual search. Cognition, 85, 37-52. 
Lambert, A., \& Hockey, R. (1986). Selective attention and performance with a multidimensional visual display. Journal of Experimental Psychology: Human Perception \& Performance, 12, 484-495.

Maljkovic, V., \& Nakayama, K. (1996). Priming of pop-out: II. The role of position. Perception \& Psychophysics, 58, 977-991.

Maljkovic, V., \& NaKayama, K. (2000). Priming of popout: III. A short-term implicit memory system beneficial for rapid target selection. Visual Cognition, 7, 571-595.

MiLleR, J. (1988). Components of the location probability effect in visual search tasks. Journal of Experimental Psychology: Human Perception \& Performance, 14, 453-471.

MüLLER, H. J., \& RabBitT, P. M. (1989). Reflexive and voluntary orienting of visual attention: Time course of activation and resistance to interruption. Journal of Experimental Psychology: Human Perception \& Performance, 15, 315-330.

Musen, G. (1996). Effects of task demands on implicit memory for object-location associations. Canadian Journal of Experimental Psychology, 50, 104-113.

Patel, G. A., \& Sathian, K. (2000). Visual search: Bottom-up or topdown? Frontiers in Bioscience, 5, D169-D193.

Peterson, M. S., \& Kramer, A. F. (2001a). Attentional guidance of the eyes by contextual information and abrupt onsets. Perception \& Psychophysics, 63, 1239-1249.

Peterson, M. S., \& Kramer, A. F. (2001b). Contextual cueing reduces interference from task-irrelevant onset distractors. Visual Cognition, 8, 843-859.

Posner, M. I. (1980). Orienting of attention. Quarterly Journal of Experimental Psychology, 32, 3-25.

Posner, M. I., Snyder, C. R., \& Davidson, B. J. (1980). Attention and the detection of signals. Journal of Experimental Psychology: General, 109, 160-174.

Reder, L. M., Weber, K., Shang, J., \& Vanyukov, P. M. (2003). The adaptive character of the attentional system: Statistical sensitivity in a target localization task. Journal of Experimental Psychology: Human Perception \& Performance, 29, 631-649.

Saffran, J. R. (2002). Constraints on statistical language learning. Journal of Memory \& Language, 47, 172-196.

Shaw, M. L., \& Shaw, P. (1977). Optimal allocation of cognitive resources to spatial locations. Journal of Experimental Psychology: Human Perception \& Performance, 3, 201-211.

Shomstein, S., \& Yantis, S. (2004). Configural and contextual prioritization in object-based attention. Psychonomic Bulletin \& Review, 11, 247-253

Theeuwes, J. (1991). Exogenous and endogenous control of attention The effect of visual onsets and offsets. Perception \& Psychophysics, 49, 83-90.

Theeuwes, J., Kramer, A. F., \& Atchley, P. (1999). Attentional effects on preattentive vision: Spatial precues affect the detection of simple features. Journal of Experimental Psychology: Human Perception \& Performance, 25, 341-347.

Treisman, A., \& Gelade, G. (1980). A feature-integration theory of attention. Cognitive Psychology, 12, 97-136.
Tse, P. U., Sheinberg, D. L., \& Logothetis, N. (2003). Attentional enhancement opposite a peripheral flash revealed using change blindness. Psychological Science, 14, 91-99.

Willingham, D. B., Salidis, J., \& Gabrieli, J. D. E. (2002). Direct comparison of neural systems mediating conscious and unconscious skill learning. Journal of Neurophysiology, 88, 1451-1460.

YANTIS, S. (1998). Control of visual attention. In H. Pashler (Ed.), Attention (pp. 223-256). Hove, UK: Psychology Press/Erlbaum.

YANTIS, S., \& EGETH, H. E. (1999). On the distinction between visual salience and stimulus-driven attentional capture. Journal of Experimental Psychology: Human Perception \& Performance, 25, 661-676.

Yantis, S., \& Jonides, J. (1990). Abrupt visual onsets and selective attention: Voluntary versus automatic allocation. Journal of Experimental Psychology: Human Perception \& Performance, 16, 121-134.

ZeLINSKY, G. J. (1999). Precuing target location in a variable set size "nonsearch" task: Dissociating search-based and interference-based explanations for set size effects. Journal of Experimental Psychology: Human Perception \& Performance, 25, 875-903.

Zelinsky, G. J., Rao, R. P. N., Hayhoe, M. M., \& Ballard, D. H. (1997). Eye movements reveal the spatiotemporal dynamics of visual search. Psychological Science, 8, 448-453.

\section{NOTE}

1. One logical question that follows from these results involves the speed with which responses to targets in the high-probability location begin to be facilitated, relative to low probability location targets within a single block. We were unable to address this question in this experiment because of the limited number of blocks per participant. However, using a similar experiment with shorter block lengths (60 as compared with 180 trials), we were able to examine each time the target reappeared in a high- or low-probability location after intervening trials in a different location. For example, in a sequence of trials such as high, high, high, low, high, the fifth trial would be labeled as the second appearance of the target in the high-probability location. First, the main effect of probability was present and showed the same pattern as that found in Experiment $1[F(2,14)=77.1, M S=611, p<.001]$. Second, in the analysis of interest, we found that the first and the third appearance of the target in the high-probability location differed significantly from each other $[t(7)=3.5, p<.01$; difference $=90.2 \mathrm{msec}]$, whereas the first and second and the second and third did not $[t(7)=1.96$; difference $=57.0 \mathrm{msec} ; t(7)=1.7$; difference $=33.1]$. Consistent with the progressive reduction of RT for high probability location targets, only the second and the third appearances differed between high and low probability location targets. This result suggested that facilitation for targets in the high-probability location was rapid and likely shares learning mechanisms with repetition priming.

(Manuscript received November 10, 2003; revision accepted for publication February 3, 2005.) 